\title{
Dynamic phase assembled terahertz metalens for reversible conversion between linear polarization and arbitrary circular polarization
}

\author{
Jitao $\mathrm{Li}^{1 \dagger}$, Guocui Wang ${ }^{2,3 \dagger}$, Zhen Yue ${ }^{1 \dagger}$, Jingyu Liu ${ }^{3}$, Jie $\mathrm{Li}^{1}$, \\ Chenglong Zheng ${ }^{1}$, Yating Zhang ${ }^{1 *}$, Yan Zhang ${ }^{3 *}$ and Jianquan Yao $^{1 *}$
}

If a metalens integrates the circular polarization (CP) conversion function, the focusing lens together with circular-polarizing lens $(\mathrm{CPL})$ in traditional cameras may be replaced by a metalens. However, in terahertz (THz) band, the reported metalenses still do not obtain the perfect and strict single-handed CP, because they were constructed via PancharatnamBerry phase so that CP conversion contained both left-handed CP (LCP) and right-handed CP (RCP) components. In this paper, a silicon based $\mathrm{THz}$ metalens is constructed using dynamic phase to obtain single-handed CP conversion. Also, we can rotate the whole metalens at a certain angle to control the conversion of multi-polarization states, which can simply manipulate the focusing for incident linear polarization (LP) THz wave in three polarization conversion states, including LP without conversion, LCP and RCP. Moreover, the polarization conversion behavior is reversible, that is, the $\mathrm{THz}$ metalens can convert not only the LP into arbitrary single-handed CP, but also the LCP and RCP into two perpendicular LP, respectively. The metalens is expected to be used in advanced THz camera, as a great candidate for traditional CPL and focusing lens group, and also shows potential application in polarization imaging with discriminating LCP and RCP.

Keywords: terahertz metalens; metamaterials; metasurfaces; dynamic phase; single-handed circular polarization; reversible conversion

Li JT, Wang GC, Yue Z, Liu JY, Li J et al. Dynamic phase assembled terahertz metalens for reversible conversion between linear polarization and arbitrary circular polarization. Opto-Electron Adv 5, 210062 (2022).

\section{Introduction}

There are a lot of linear polarization (LP) light in nature, and many LP light are harmful in photography. They will introduce reflection light into the camera to reduce the clarity and bright-dark contrast of the photo. Polarizer can filter out these harmful LP light, making the color of the photo more saturated and clear. Ordinary cameras usually use a linear-polarizing lens and rotate it to reduce LP light incidence. However, there is still an ignorable remnant of LP light within camera, which still cause

${ }^{1}$ Key Laboratory of Opto-Electronics Information Technology (Tianjin University), Ministry of Education, School of Precision Instruments and Opto-Electronics Engineering, Tianjin University, Tianjin 300072, China; ${ }^{2}$ Beijing Engineering Research Center for Mixed Reality and Advanced Display, School of Optics and Photonics, Beijing Institute of Technology, Beijing 100081, China; ${ }^{3}$ Beijing Key Laboratory for Metamaterials and Devices, Key Laboratory of Terahertz Optoelectronics, Ministry of Education, and Beijing Advanced Innovation Center for Imaging Technology, Department of Physics, Capital Normal University, Beijing 100048, China.

tThese authors contributed equally to this work.

*Correspondence: YT Zhang, E-mail: yating@tju.edu.cn; Y Zhang, E-mail: yzhang@cnu.edu.cn; JQ Yao, E-mail: jqyao@tju.edu.cn Received: 12 May 2021; Accepted: 21 July 2021; Published online: 25 January 2022

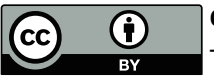

Open Access This article is licensed under a Creative Commons Attribution 4.0 International License.

To view a copy of this license, visit http://creativecommons.org/licenses/by/4.0/.

(C) The Author(s) 2022. Published by Institute of Optics and Electronics, Chinese Academy of Sciences. 
some harmful results. In some advanced cameras with auto focus (AF) and automatic exposure (AE), the residual LP light inside the camera will make AF invalid and $\mathrm{AE}$ inaccurate. In this case, camera has to depend on the circular-polarizing lens (CPL), which can directly convert the incident $\mathrm{LP}$ light into circular polarization $(\mathrm{CP})$ light. For most optical devices, the $\mathrm{CP}$ light has no difference with the non-polarized light. Theoretically, the CPL can be used in any camera, including advanced terahertz $(\mathrm{THz})$ camera. However, the preparation of the traditional CPL still depends on manual polishing and sanding, so that the CPL is expensive, low yields and generally large body that is difficult to integrate.

In recent years, researchers have devoted themselves to developing a novel polarization conversion device with lower cost, smaller size, higher integration degree and mutifunctions. Metamaterials/metasurfaces provide a good solution for these new devices ${ }^{1-6}$. The $\mathrm{THz} \mathrm{CP}$ conversion devices based on metasurfaces have been developed ${ }^{7}$. Further, to give full play to the advantages of the metasurfaces, the wavefront control devices combined with phase operation are realized, such as metalens ${ }^{8-21}$. By arranging different structure units according to a certain phase distribution, the metalens can realize not only the focusing function similar to the traditional convex lens, but also the polarization conversion function ${ }^{22-26}$. In general, $\mathrm{THz}$ metalens widely uses all-dielectric silicon $(\mathrm{Si})$ material with transmission structure so as to meet the practical application $\mathrm{s}^{27}$. If $\mathrm{THz}$ metalens can also achieve CP conversion, they can replace both traditional CPL and focus lens, and are used in advanced $\mathrm{THz}$ camera, further reducing the camera volume. A very important performance index is that metalens must perfectly convert incident LP light into single-handed CP light, which means the converted CP light can not contain both the left-handed and the righthanded. In the aspect of polarization conversion, the existing $\mathrm{THz}$ metalenses have realized the LP rotation ${ }^{22,25}$, but the polarization conversion of LP to single-handed $\mathrm{CP}$ is not realized. Using the chiral optics and the spin decoupling mechanisms to construct $\mathrm{THz}$ metalenses may obtain the conversion and focusing of $\mathrm{LP}$ to $\mathrm{CP}^{28-30}$. However, the physical principles of these techniques involves the separation of left-handed circular polarization (LCP) and right-handed circular polarization (RCP) from incident LP, which does not allow the clean and efficient conversion of LP to single-handed CP. In other words, $\mathrm{CP}$ conversion in those $\mathrm{THz}$ metalenses contains both LCP and RCP components, and it is impossible to completely eliminate one $\mathrm{CP}$ but retain the opposite one. As a result, a part of LCP and RCP waves may combine into harmful LP waves again, so that those metalenses cannot still be applied in advanced $\mathrm{THz}$ camera.

The metalens must depend on several different structure units those phase can cover $0-2 \pi$. Traditionally, the principle of Pancharatnam-Berry $(\mathrm{PB})$ phase is in whole or in part introduced in the phase design of the $\mathrm{THz}$ metalens, even for those $\mathrm{THz}$ metalenses containing chiral optics and spin decoupling mechanisms ${ }^{28-30}$. For instance, the general operation is that several basic structures with different structural parameters are firstly designed to meet the phase change of $0-\pi$, and then the phases of the remaining $\pi-2 \pi$ are obtained by rotating these structures at $90^{\circ}$. Assuming that a basic structure unit can realize the conversion of LP to single-handed $\mathrm{CP}$, the helix of $\mathrm{CP}$ will be opposite direction after this structure being rotated by $90^{\circ}$ due to the spin dependence of $\mathrm{PB}$ phase, which eventually results in the failure of conversion and focusing of LP to single-handed CP in whole metalens. Hence, these intrinsic shortcomings of PB phase in physical principle lead to that the $\mathrm{THz}$ metalens integrating the polarization conversion function of LP to single-handed CP has not been realized yet.

Meanwhile, the polarization conversion of the existing metalenses is usually not reversible. Although a LP $\mathrm{THz}$ wave through those metalenses is converted into other polarization states and focused, $\mathrm{THz}$ wave with other polarization states through those metalenses can no longer be converted into the original LP and focused. These shortcomings also limit the multifunctional applications of the $\mathrm{THz}$ metalens to some extent.

To overcome mentioned shortcomings in polarization conversion of the existing $\mathrm{THz}$ metalenses and expand their multifunctional applications, we designed an all-dielectric $\mathrm{Si}$ based $\mathrm{THz}$ metalens, which was assembled using dynamic phase (relating to the structural parameters of structure units) and getting rid of the $\mathrm{PB}$ phase (relating to rotation of structure units). Some new features appear and are described in Fig. 1. (i) The LP $\mathrm{THz}$ wave through metalens will be completely converted into a single-handed CP and then focused at a certain time. Next, we only need to rotate this metalens with $\pm 45^{\circ}$ and $\pm 90^{\circ}$ to achieve arbitrary switching among LP, LCP and RCP, as shown in Fig. 1(a-b). This feature shows it can be used in advanced $\mathrm{THz}$ camara, as a candidate to replace the group of traditional CPL and 

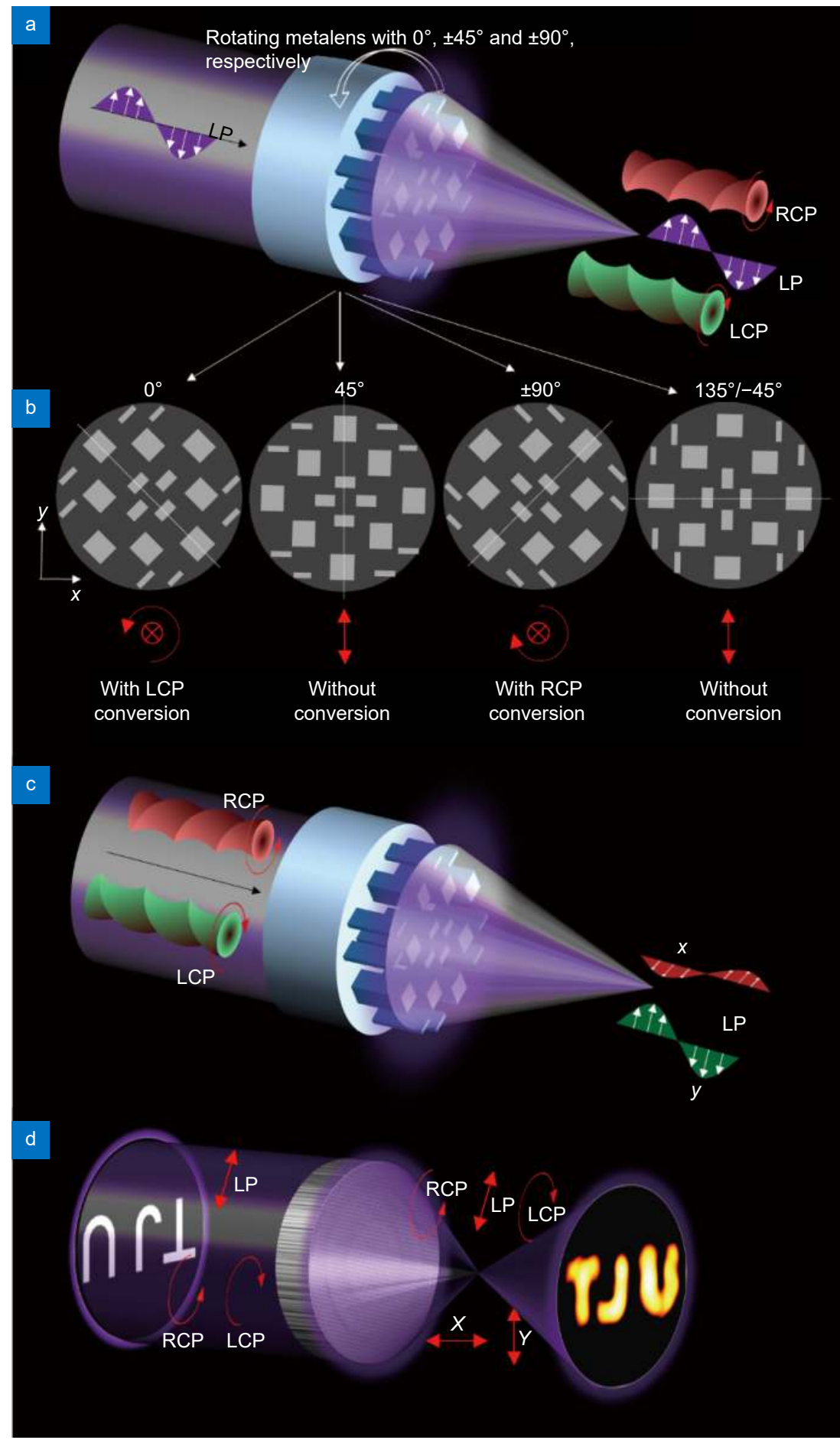

Fig. 1 | (a, b) With incident LP, the schematic functions for conversion and focusing of the metalens with different rotation angles. (c) With incident LCP and RCP, the schematic functions for conversion and focusing of the metalens. (d) The schematic THz imaging. The helix directions of LCP and RCP waves correspond to counterclockwise and clockwise rotation of electric field vector when viewed along wave propagation direction, respectively.

focusing lens. (ii) The polarization conversion is reversible, as shown in Fig. 1(c). When a single-handed CP $\mathrm{THz}$ wave passes through the metalens, it will be completely converted into LP and focused. Moreover, incident RCP and LCP correspond to two transmission LP waves which are always perpendicular to each other. When this property is used for polarization imaging, the incident RCP and LCP THz waves can be distinguished easily by monitoring transmission LP waves in the image plane, as shown in Fig. 1(d). The polarization 
imaging function may has a potential application in some fields such as chiral material studies.

\section{Experiments and methods}

The metalens model was built in commercial CST Microwave Studio software, and all numerical simulation results were obtained by time-domain solver. The simple silicon ( $\mathrm{Si}$ ) rectangular bar is used as basic structure to construct the metalens, and the structural parameters (height, length and width of Si rectangular bar) of units are selected from a large number of parameter scanning results. The metalens sample used high resistance $\mathrm{Si}$ wafer (conductivity $\delta<0.05 \mathrm{~S} \mathrm{~m}^{-1}$ ) with $500 \mu \mathrm{m}$ thickness, and was prepared using plasma etching method (ICP ion etching machine with $\mathrm{SF}_{6}$ etching gas). The characterization system is shown as Fig. 2. A femtosecond laser outputs a 50 fs duration pulse with $800 \mathrm{~nm}$ central wavelength, and it goes through the beam splitter L1 to be separated into a probe beam and a pump beam. The slender pump beam reflected by the mirror L2 is firstly expanded via a concave lens L3 to cover enough the surface $(1 \mathrm{~cm} \times 1 \mathrm{~cm})$ of $<110>\mathrm{ZnTe}$ crystal, and then induces optical rectification effects to radiate the linear polarization $\mathrm{THz}$ wave ${ }^{31}$. Using a metallic parabolic mirror L4 collimate the $\mathrm{THz}$ beam before sample being irradiated by $\mathrm{THz}$ wave. $\mathrm{THz}$ wave through sample is received by a $\mathrm{ZnTe}$ detection crystal. At the same time, the slender probe beam is also expanded by a beam expanding system consisting of a series of lenses, and then adjust probe polarization through a half wave plate (HWP) and a polarizer. The probe beam will be reflected onto the detection crystal by the beam splitter L5. Subsequently, the received $\mathrm{THz}$ field excites Pockels effect in
ZnTe detection crystal to modulate the polarization of probe beam. Then, the probe beam with polarization modulation through L5 is acquired by CCD camera to form eventually a $\mathrm{THz}$ image based on a balanced electro-optics detection technique ${ }^{32}$. This detection process is essentially the electro-optical sampling ${ }^{33}$, and this system includes two convex lenses L6 and L7, a quarter wave plate (QWP) and a Wollaston prism (PBS). Besides, it is required to synchronously control the mechanical chopper and CCD camera for using a dynamics subtraction method to eliminate the background intensity of the probe beam.

\section{Results and discussion}

\section{Conversion and focusing of LP to arbitrary single- handed CP}

The simple Si rectangular bar is used as basic structure to design metalens. Started by a feature that the incident $x$ LP $\mathrm{THz}$ wave through metalens is converted into LCP and focused, we discuss firstly the design process of metalens and then deduce other features.

At the first, let us see the polarization conversion property of a rectangular bar. After incident electric field through Si rectangular bar, the universal formation of transmission electric field is as follow:

$$
\left(\begin{array}{c}
\boldsymbol{E}_{x}^{\mathrm{t}} \\
\boldsymbol{E}_{y}^{\mathrm{t}}
\end{array}\right)=\left(\begin{array}{cc}
t_{x x} & t_{x y} \\
t_{y x} & t_{y y}
\end{array}\right)\left(\begin{array}{c}
\boldsymbol{E}_{x}^{\mathrm{i}} \\
\boldsymbol{E}_{y}^{\mathrm{i}}
\end{array}\right)=\boldsymbol{T}\left(\begin{array}{c}
\boldsymbol{E}_{x}^{\mathrm{i}} \\
\boldsymbol{E}_{y}^{\mathrm{i}}
\end{array}\right),
$$

where $\boldsymbol{E}_{x}^{\mathrm{i}}, \boldsymbol{E}_{y}^{\mathrm{i}}$ are incident electric field at $x$-axis and $y$-axis, respectively, and $\boldsymbol{E}_{x}^{\mathrm{t}}, \boldsymbol{E}_{y}^{\mathrm{t}}$ are transmission electric field at $x$-axis and $y$-axis, respectively. $T$ is the transfer Jones matrix and consists of transmission coefficients $t_{a b}(a$, $b \in\{x, y\}, t_{a b}$ means the coefficient of transmission $a$-LP

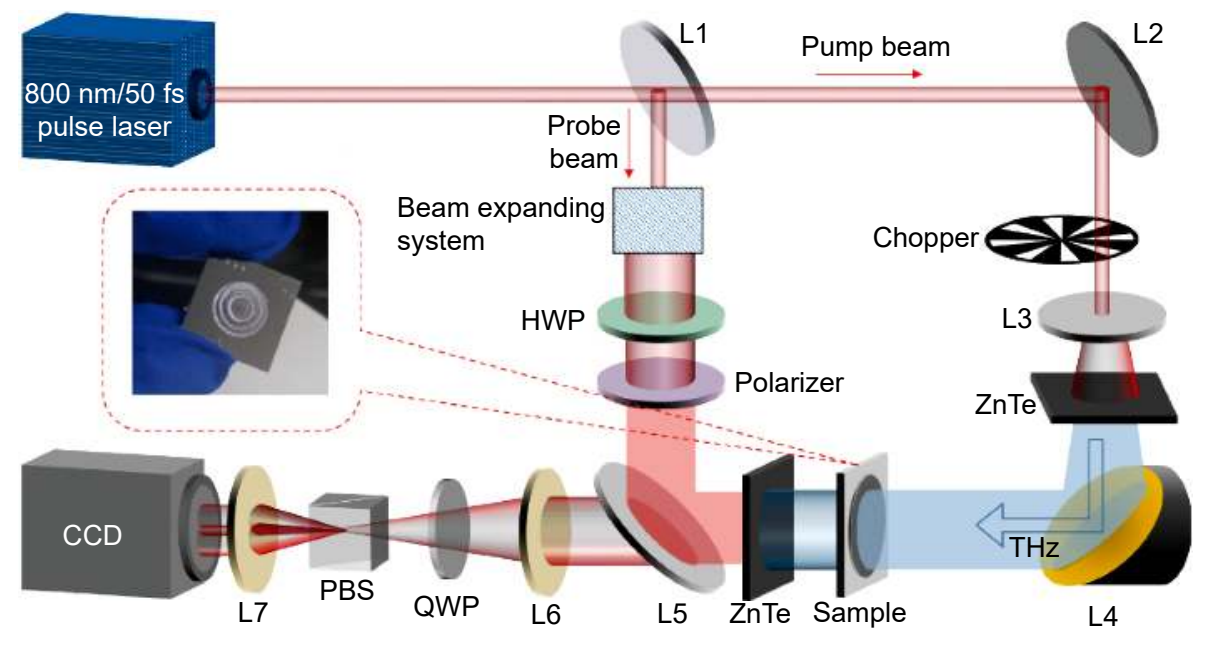

Fig. 2 | The schematic characterization system for THz focusing metalens. 

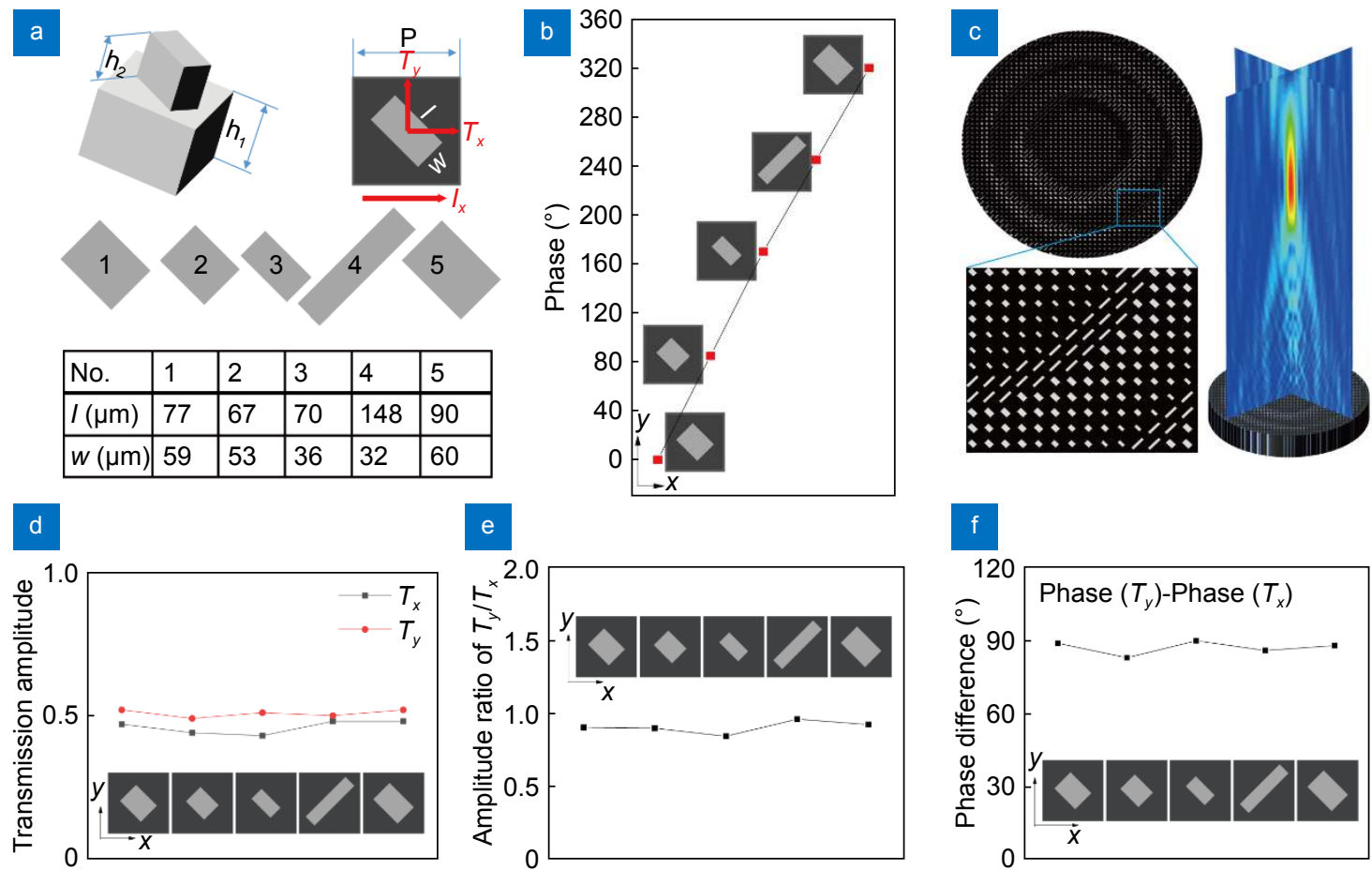

Fig. 3 | (a) The schematic five basic structures, and parameters of length ' $"$ ' and width ' $w$ ' shown in inset table; $I_{x}$ : incident $x$-LP, $T_{x}$ : co-polarization transmission at $x$-axis, $T_{y}$ : cross-polarization transmission at $y$-axis. (b) The phases based on $T_{x}$ of five basic structures. (c) The schematic picture of the metalens. (d) The amplitude of $T_{x}$ and $T_{y}$ under incident $x$-LP. (e) The amplitude ratio of $T_{y} / T_{x}$. (f) The phase difference between $T_{x}$ and $T_{y}$.

under incident $b$-LP). To obtain LCP conversion under incident $x$-LP wave (electric field vector $\left.\left(\begin{array}{ll}1 & 0\end{array}\right)^{\mathrm{T}}\right)$, the initial site of Si rectangular bar is deduced to $45^{\circ}$ deviated from $x$-axis, as shown in Fig. 3(a). The deduction process is shown in Section 1 of Supplementary Information in detail. The transmission LCP wave $\boldsymbol{E}_{0}^{\mathrm{t}}$ and transfer Jones matrix $\boldsymbol{T}_{\mathbf{0}}$ is expressed as follow:

$$
\begin{gathered}
\boldsymbol{T}_{0}=\beta\left(\begin{array}{cc}
1 & -\mathrm{i} \\
-\mathrm{i} & 1
\end{array}\right), \\
\boldsymbol{E}_{0}^{\mathrm{t}}=\boldsymbol{T}_{0}\left(\begin{array}{l}
1 \\
0
\end{array}\right)=\beta\left(\begin{array}{c}
1 \\
-\mathrm{i}
\end{array}\right),
\end{gathered}
$$

where $\beta$ is a coefficient (see section S1). The Si substrate is $h_{1}=300 \mu \mathrm{m}$, the height of Si bar is $h_{2}=200 \mu \mathrm{m}$, and the side length of square unit cell is $P=160 \mu \mathrm{m}$. An $\mathrm{x}$-LP wave through this unit cell will split into a co-polarization component $\left(T_{x}\right)$ and a cross-polarization component $\left(T_{y}\right)$. The two transmission components $\left(T_{x}\right.$ and $\left.T_{y}\right)$ perpendicular to each other have a phase difference of $\pi / 2$ and the same amplitude (about 0.5 ), which composes eventually a LCP wave. If the geometric parameters at length and width of Si bar are changed, which will obtain the phase variations of $0-2 \pi$. Five basic structures are found, and their shapes and parameters are as shown in Fig. 3(a). The phases (based on $T_{x}$ ) of five basic struc- tures vary generally from 0 to $2 \pi$, as shown in Fig. 3(b). Further, using five basic structures to construct an array meeting phase profile in Eq. (4) can obtain $\mathrm{THz}$ metalens, as shown in Fig. 3(c).

$$
\phi=\frac{2 \pi}{\lambda}\left(f-\sqrt{x^{2}+y^{2}+f^{2}}\right),
$$

where the wavelength $\lambda$ corresponds to $0.96 \mathrm{THz},(x, y)$ belongs to the coordinates of any point in this metalens with setting the origin point $(0,0)$ at center of metalens, and $f=8 \mathrm{~mm}$ is the focal length of metalens. Meanwhile, to realize complete and stable LCP conversion, almost the same amplitude and stable phase difference of $\pi / 2$ are required for two components $\left(T_{x}\right.$ and $T_{y}$ ) of every $\mathrm{Si}$ bar, as shown in Fig. 3(d-f).

Since the phase variations of $0-2 \pi$ in metalens are achieved by changing structural parameters of Si bars and do not depend on rotation operation of Si bars, this is a dynamic phase assembled $\mathrm{THz}$ metalens. Obviously, if any $\mathrm{PB}$ phases is introduced in metalens (i.e., traditionally, the phase variations of $0-\pi$ in metalens are achieved by changing structural parameters of Si bars, but the phase variations of $\pi-2 \pi$ are got by $90^{\circ}$ rotation operation of the existing Si bars), the polarization conversion of metalens will be complex so that the focusing with 
pure LCP conversion fails.

Next, we deduce other features of the $\mathrm{THz}$ metalens based on Jones matrix (Eq. (2)) of conversion from $x$-LP to LCP. When the metalens is rotated as a whole, with angles of $\theta=0^{\circ}, 45^{\circ}, 90^{\circ}, 135^{\circ}$, respectively, every unit cell will be rotated correspondingly as shown in Fig. 4(a). Respectively, new transfer Jones matrices are deduced in Section 2 of Supplementary Information and shown as follows:

For $\theta=45^{\circ}, \boldsymbol{T}_{1}=\sqrt{2} \beta\left(\begin{array}{cc}1+\mathrm{i} & 0 \\ 0 & 1-\mathrm{i}\end{array}\right)$.

For $\theta=90^{\circ} /-90^{\circ}, \boldsymbol{T}_{2}=\beta\left(\begin{array}{cc}1 & \mathrm{i} \\ \mathrm{i} & 1\end{array}\right)$.

For $\theta=135^{\circ} /-45^{\circ}, \boldsymbol{T}_{3}=\sqrt{2} \beta\left(\begin{array}{cc}1-\mathrm{i} & 0 \\ 0 & 1+\mathrm{i}\end{array}\right)$.

Correspondingly, the electric field of transmission waves under incident $x$-LP are expressed as:

For $\theta=45^{\circ}, \boldsymbol{E}_{1}^{\mathrm{t}}=\boldsymbol{T}_{1}\left(\begin{array}{l}1 \\ 0\end{array}\right)=\sqrt{2} \beta(1+\mathrm{i})\left(\begin{array}{l}1 \\ 0\end{array}\right)$.

For $\theta=90^{\circ} /-90^{\circ}, \boldsymbol{E}_{2}^{\mathrm{t}}=\boldsymbol{T}_{2}\left(\begin{array}{l}1 \\ 0\end{array}\right)=\beta\left(\begin{array}{l}1 \\ \mathrm{i}\end{array}\right)$.

For $\theta=135^{\circ} /-45^{\circ}, \boldsymbol{E}_{3}^{\mathrm{t}}=\boldsymbol{T}_{3}\left(\begin{array}{l}1 \\ 0\end{array}\right)=\sqrt{2} \beta(1-\mathrm{i})\left(\begin{array}{l}1 \\ 0\end{array}\right)$.

Equations (8) and (10) mean that when the metalens is rotated with $\pm 45^{\circ}$, the transmission waves are still $x$-LP without polarization conversion. Also, five basic structures show almost the same transmission amplitude, as shown in Fig. 4(b, d). At this moment, the metalens will focus $x$-LP without polarization conversion. When the metalens is rotated with $\pm 90^{\circ}$, formula (9) show that the transmission RCP conversion will appear. Obviously, the transmission amplitude of two components $\left(T_{x}\right.$ and $\left.T_{y}\right)$ are same as that before rotating metalens, but two components $\left(T_{x}\right.$ and $T_{y}$ ) will have a phase difference of $\Delta \varphi=-\pi / 2$ as shown in Fig. 4(c), meaning the metalens will convert incident $x$-LP into RCP and then focus.

The above analyses indicate that we only need to rotate this metalens with $\pm 90^{\circ}$ to focus an incident $x$-LP with arbitrary single-handed CP conversion, respectively. In fact, it is easy to understand that arbitrary LP in $x$-o- $y$ plane through metalens can also be focused with arbitrary single-handed CP conversion. For instance, if the incident LP has an angle $\phi$ to $x$-axis, we can rotate this metalens with an additional angle $\phi$ on basis of $\theta$ rotation angle. Then, this will have the same meaning as the above. Hence, the focusing and conversion of arbitrary LP to arbitrary single-handed CP can be obtained. Here, we can give their general forms. The incident LP
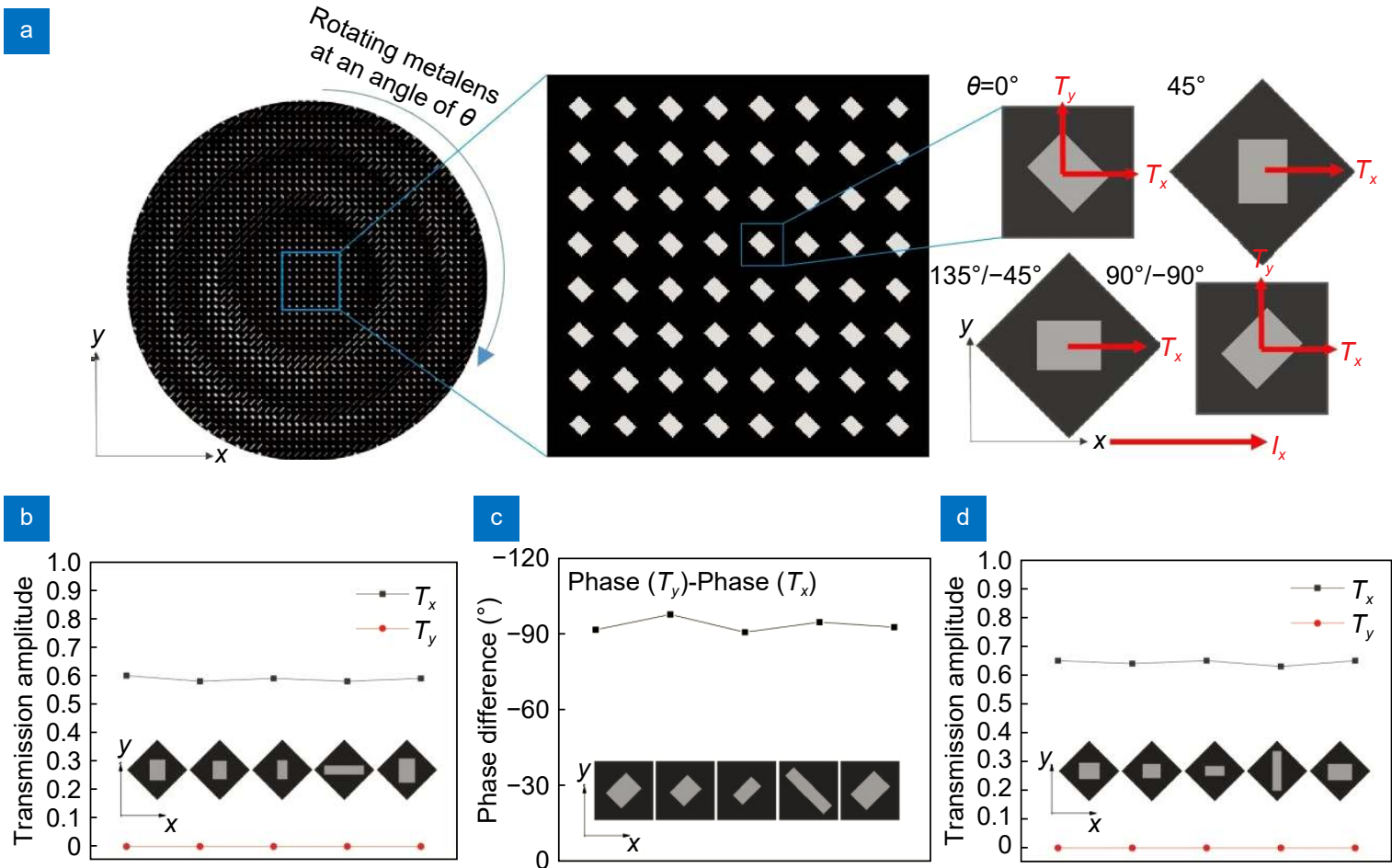

Fig. 4 | (a) The schematic change of unit cells when the whole metalens is rotated with $\theta$. (b) When the whole metalens is rotated with $45^{\circ}$, the amplitude of $T_{x}$ and $T_{y}$ under incident $x$-LP. (c) When the whole metalens is rotated with $\pm 90^{\circ}$, the phase difference of $T_{x}$ and $T_{y}$ under incident $x$ LP. (d) When the whole metalens is rotated with $135^{\circ} /-45^{\circ}$, the phase difference of $T_{x}$ and $T_{y}$ under incident $x$-LP. 
with arbitrary direction in $x-o-y$ plane can be obtained by rotating $x$-LP at an angle of $\phi$, thus incident arbitrary LP wave $\boldsymbol{E}_{A}^{i}$ in $x-o-y$ plane can be expressed via a base matrix $\boldsymbol{H}$ as follow:

$\boldsymbol{E}_{A}^{\mathrm{i}}=\boldsymbol{H}\left(\begin{array}{l}1 \\ 0\end{array}\right)=\left(\begin{array}{rr}\cos \varphi & -\sin \varphi \\ \sin \varphi & \cos \varphi\end{array}\right)\left(\begin{array}{l}1 \\ 0\end{array}\right)=\left(\begin{array}{c}\cos \varphi \\ \sin \varphi\end{array}\right)$.

Further, the metalens should be rotated with an additional angle $\phi$ on basis of $\theta$ rotation angle, and new transfer Jones matrix is:

$$
\begin{gathered}
\boldsymbol{T}_{n}^{\prime}=\boldsymbol{H}_{n} \boldsymbol{H}^{-1}=\left(\begin{array}{rr}
\cos \varphi & -\sin \varphi \\
\sin \varphi & \cos \varphi
\end{array}\right) \boldsymbol{T}_{n}\left(\begin{array}{rr}
\cos \varphi & \sin \varphi \\
-\sin \varphi & \cos \varphi
\end{array}\right) \\
n=0,1,2,3
\end{gathered}
$$

At this moment, this situation is equivalent to the an $x$-LP through the metalens without additional rotation angle $\phi$. Eventually, the transmission electric field is expressed:

$$
\begin{gathered}
\boldsymbol{E}_{n}^{\mathbf{t}^{\prime}}=\boldsymbol{T}_{n}^{\prime} \boldsymbol{E}_{\boldsymbol{A}}^{\mathrm{i}}=\left(\begin{array}{rr}
\cos \varphi & -\sin \varphi \\
\sin \varphi & \cos \varphi
\end{array}\right) \boldsymbol{E}_{n}^{\mathrm{t}}=\boldsymbol{H}_{n}^{\mathrm{t}}, \\
n=0,1,2,3 .
\end{gathered}
$$

It can be seen from formula (13) that the final transmission electric field $\boldsymbol{E}_{n}^{\mathbf{t}^{\prime}}$ is equivalent to rotating the previous transmission electric field $\boldsymbol{E}_{n}^{\mathrm{t}}$ with an additional angle $\phi$. The detailed deduction process for Eqs. (11-13) can be seen in Section 3 of Supplementary Information.

The SEM of original metalens is shown in Fig. 5(a). And Fig. 5(b) shows simulative and experimental results in focal plane for focusing and conversion of incident $x$ LP to transmission CP. In general, these experimental results are well agreement with the simulative results. The original metalens without any rotation $\left(\theta=0^{\circ}\right)$ possesses a function of converting $x$-LP into pure LCP. When metalens is rotated at $\theta= \pm 90^{\circ}, x$-LP to pure RCP conversion and focusing can be obtained. Further, if we want to keep $x$-LP focusing without polarization conversion, the metalens can be rotated at $\theta= \pm 45^{\circ}$, as shown in Fig. 5(c). Overall, the metalens we designed has some practical values, including simple operation, high integration, and easily obtaining arbitrary single-handed CP.

\section{Conversion and focusing of arbitrary single-handed CP to LP}

The reversible polarization conversion is another characteristic of this metalens, that is, the metalens can convert not only incident LP into single-handed CP, but also incident single-handed CP into LP. For original metalens without any rotation $\left(\theta=0^{\circ}\right)$, the incident RCP and LCP are transferred by Jones matrix $\boldsymbol{T}_{0}$, and transmission fields $\boldsymbol{E}_{n}^{t}(n=4,5)$ as follows:

$$
\boldsymbol{E}_{4}^{\mathrm{t}}=\boldsymbol{T}_{0}\left(\begin{array}{l}
1 \\
\mathrm{i}
\end{array}\right)=\beta\left(\begin{array}{cc}
1 & -\mathrm{i} \\
-\mathrm{i} & 1
\end{array}\right)\left(\begin{array}{l}
1 \\
\mathrm{i}
\end{array}\right)=2 \beta\left(\begin{array}{l}
1 \\
0
\end{array}\right) .
$$

$\boldsymbol{E}_{5}^{\mathrm{t}}=\boldsymbol{T}_{0}\left(\begin{array}{c}1 \\ -\mathrm{i}\end{array}\right)=\beta\left(\begin{array}{cc}1 & -\mathrm{i} \\ -\mathrm{i} & 1\end{array}\right)\left(\begin{array}{c}1 \\ -\mathrm{i}\end{array}\right)=-2 \beta\left(\begin{array}{l}0 \\ 1\end{array}\right)$.

The above two formulas show that RCP through original metalens will be converted into an $x$-LP and focused, while LCP through this metalens will be converted into a $y$-axis LP ( $y$-LP) and focused.

Further, we can rotate the metalens to change direction of transmission LP. Here, we have also deduced their general forms in Section 5 of Supplementary Information, and results are given as follows. Assuming that metalens is rotated with an angle $\theta$, then RCP through metalens will be converted into a LP having an angle $\theta$ to $x$-axis with an additional phase shift $\theta$, as shown in Fig. 6(a), and the general form is expressed as follow:

$$
\boldsymbol{E}_{4}^{\mathbf{t}^{\prime}}=2 \beta \mathrm{e}^{\mathrm{i} \theta}\left(\begin{array}{c}
\cos \theta \\
\sin \theta
\end{array}\right) .
$$

While LCP through metalens will be converted into a LP having an angle $\theta$ to $y$-axis with an additional phase shift $(\pi / 2-\theta)$, as shown in Fig. $6(\mathrm{~b})$, and the general form is expressed as follow:

$$
\boldsymbol{E}_{5}^{\mathrm{t}^{\prime}}=-2 \beta \mathrm{e}^{\mathrm{i}\left(\frac{\pi}{2}-\theta\right)}\left(\begin{array}{r}
-\sin \theta \\
\cos \theta
\end{array}\right) .
$$

In other words, to obtain conversion and focusing of arbitrary CP to arbitrary LP, we only need to rotate the metalens at a corresponding angle of target LP. Meanwhile, it is easy to understand that no matter how many angles the metalens rotates, the transmission LP waves corresponding to incident RCP and LCP are always perpendicular to each other. To verify the mentioned theory analyses, Fig. 6(c) shows simulative and experimental results for focusing and conversion of incident CP to transmission LP. Because the transmission LP before and after metalens rotation has a corresponding relationship, we only need to present the results in term of original metalens $\left(\theta=0^{\circ}\right)$ here. It can be seen from Fig. $6(\mathrm{c})$ that the original metalens has the function of converting RCP into $y$-LP and converting LCP into $x$-LP, which verifies the mentioned theory analyses well.

\section{THz imaging applications}

The imaging results of $\mathrm{THz}$ focusing metalens are 

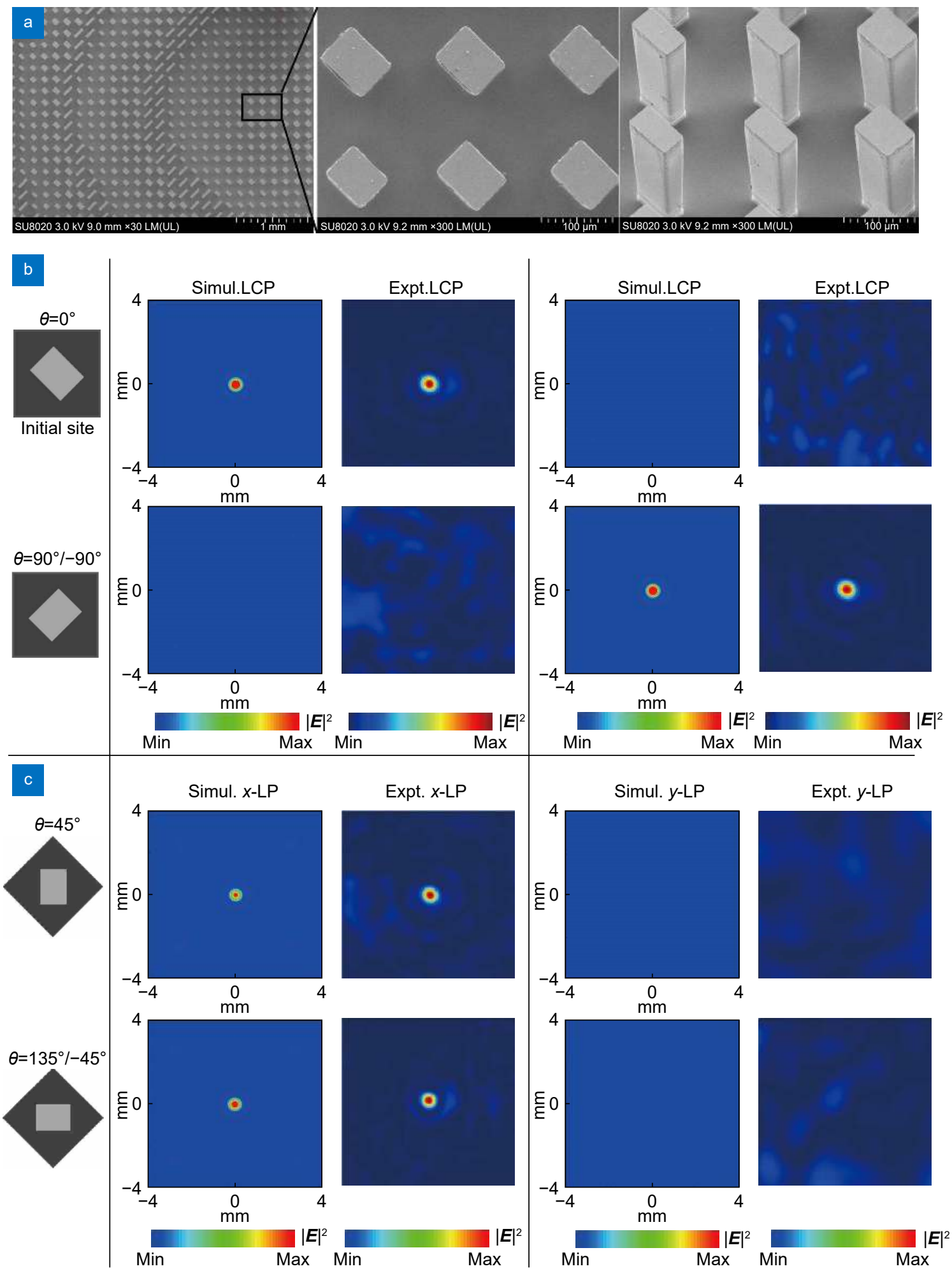

Fig. 5 | (a) The SEM images of the metalens sample. (b) The simulative and experimental results for focusing and conversion of incident $x$-LP to transmission CP. (c) The simulative and experimental results for focusing of incident $x$-LP, without polarization conversion. The simulation results in the cross section are shown in Section 4 of Supplementary information.

simulated. As long as a certain object-image relationship is satisfied, the ' $\mathrm{TJU}$ ' pattern can be presented in image plane. As shown in Fig. 7(a), the object-image relationship is $1 / f=1 / u+1 / v$, where $f=8 \mathrm{~mm}$ is focal length, and $u=v=16 \mathrm{~mm}$ are object distance and image distance, respectively. We simulate firstly the $\mathrm{CP}$ imaging properties of metalens under incident $x$-LP, as shown Fig. 7(b). Due to LCP conversion, the original metalens possesses LCP 

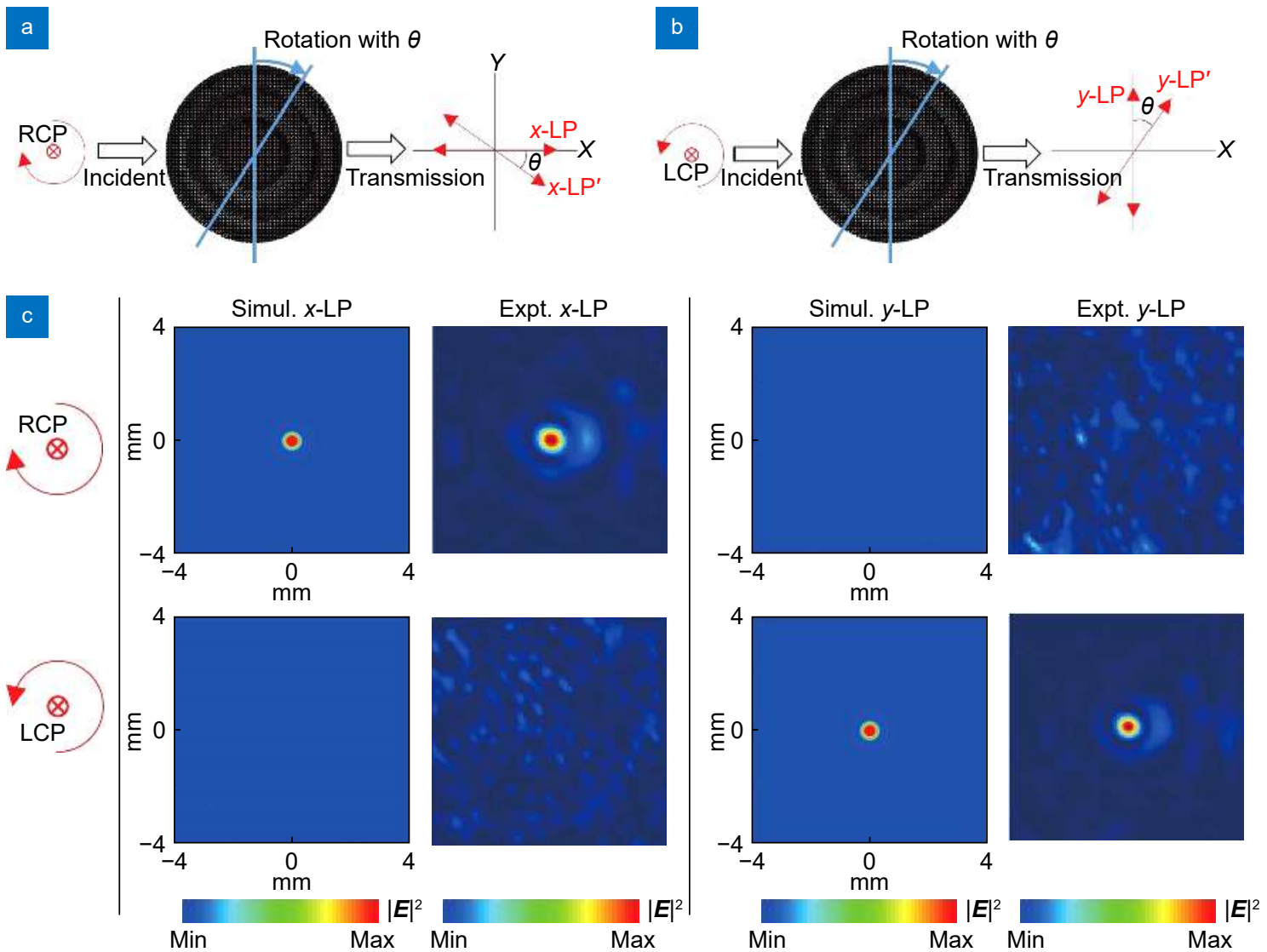

Fig. 6 | (a, b) Respectively, RCP and LCP through the rotated metalens will be converted into a LP having an angle $\theta$ to $x$-axis and $y$-axis; $x$-LP and $y$-LP corresponding to the case without metalens rotation; $x$-LP' and $y$-LP' corresponding to the case with metalens rotation. (c) The simulative and experimental results for focusing and conversion of incident $C P$ to transmission LP. The simulation results in the cross section are shown in Section 6 in Supplementary Information.

imaging property well while there is no RCP imaging property. In contrast, since the RCP conversion appears as rotating metalens at $\pm 90^{\circ}$, the metalens will present well RCP imaging property while there is no LCP imaging property. These results reveal that this metalens can be used in advanced $\mathrm{THz}$ camera, as a great candidate of traditional CP conversion and focusing device group.

In some fields, it is necessary to distinguish RCP and LCP. For instance, many natural and artificial materials such as chiral molecules can emit LCP wave (or induce LCP conversion), which are called left-handed materials, and vice versa are called right-handed materials. If the spin direction of these CP waves cannot be detected, the chiral properties of materials can not be distinguished. Here, this metalens we designed may provide a useful idea to distinguish LCP and RCP. It has been proposed in conversion and focusing of arbitrary single-handed CP to LP section that no matter how to rotate metalens, two transmission LP waves under incident RCP and LCP are always perpendicular to each other, which means two transmission LP waves have no overlapping components at all. Hence, incident RCP and LCP can be distinguished completely in polarization states by monitoring different transmission LP states. As shown in Fig. 7(c), the simulation show the original metalens under incident RCP can obtain $x$-LP imaging property well but no $y$ LP imaging property. Further, $y$-LP imaging property can be obtained under incident LCP, without any $x$-LP imaging property. These results indicate that this metalens may also be applied in polarization imaging field with LCP and RCP discrimination.

\section{Conclusions}

In this paper, to construct a metalens integrating singlehanded CP conversion, an all-dielectric $\mathrm{Si}$ based $\mathrm{THz}$ metalens was designed using dynamic phase, without any $\mathrm{PB}$ phase. Simulative and experimental results show that $\mathrm{LP} \mathrm{THz}$ wave through metalens can be converted into arbitrary single-handed $\mathrm{CP} \mathrm{THz}$ wave and then focused. As a fixed incident $x$-LP, when this metalens is rotated with $\pm 45^{\circ}$ and $\pm 90^{\circ}$, three transmission 


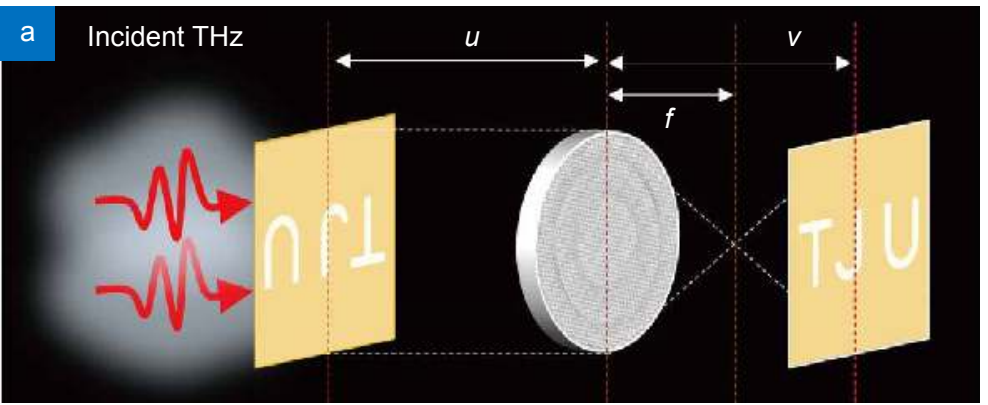

b

LCP
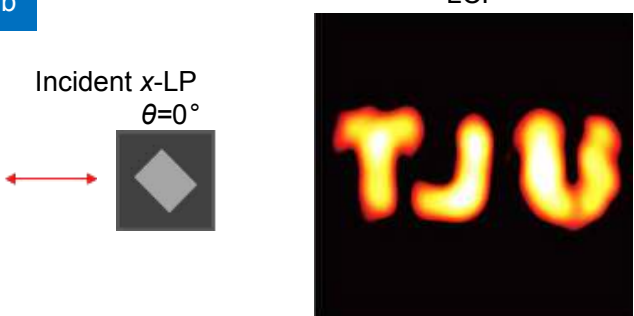

Incident $x$-LP
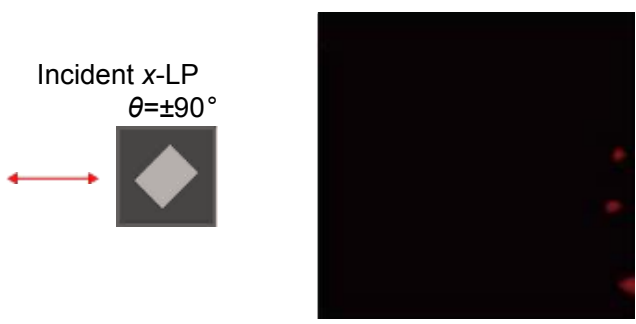

$x$-LP
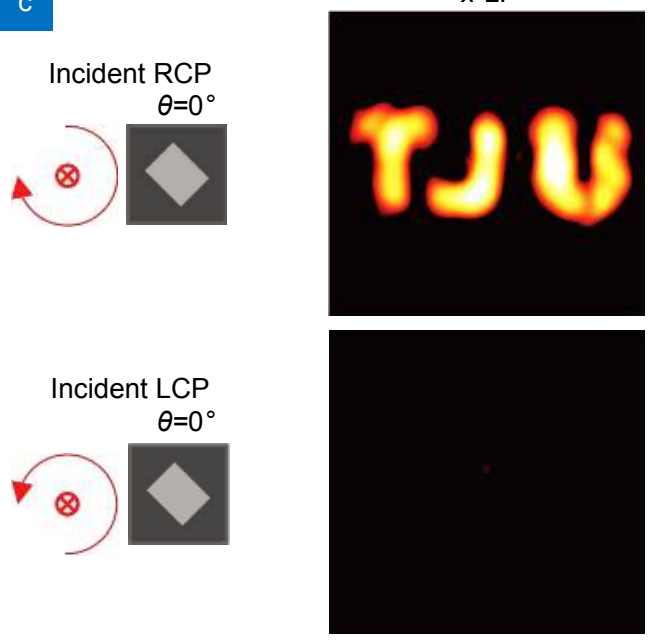

$\mathrm{RCP}$
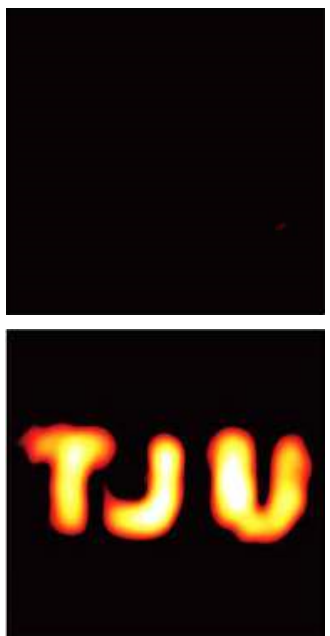

$y$-LP
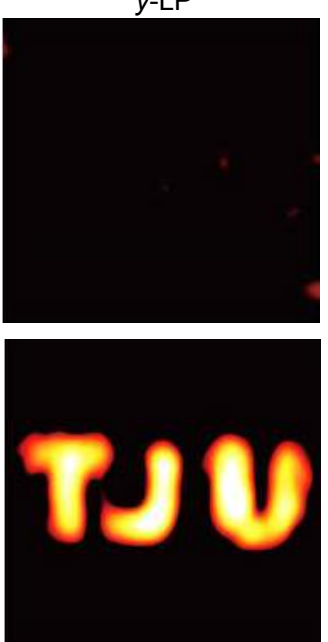

Fig. 7 | (a) The object-image relationship. (b) The simulative results for THz imaging under incident $x$-LP. (c) The simulative results for THz imaging under incident RLP and LCP.

polarization states can be obtained, including LP without conversion, LCP and RCP. Meanwhile, the polarization conversion is reversible. The arbitrary single-handed CP $\mathrm{THz}$ wave through the metalens can also be converted into LP THz wave and then focused. Respectively, RCP and LCP through the metalens are converted into $x$-LP and $y$-LP. And no matter how many angles the metalens rotates, the corresponding two transmission LP waves under incident RCP and LCP are always perpendicular to each other. Further, the $\mathrm{THz}$ imaging properties of the metalens are also verified by simulation. Results show that the metalens can be expected to become a great candidate of CP conversion and focusing device in advanced THz camera. Moreover, the metalens can be used in polarization imaging field requiring LCP and RCP discrimination, such as chiral material studies, in which the 
incident RCP and LCP will be distinguished easily by monitoring transmission LP states in the image plane.

\section{References}

1. Wu PC, Tsai WY, Chen WT, Huang YW, Chen TY et al. Versatile polarization generation with an aluminum plasmonic metasurface. Nano Lett 17, 445-452 (2017).

2. Cheng YZ, Fan JP, Luo H, Chen F. Dual-band and high-efficiency circular polarization convertor based on anisotropic metamaterial. IEEE Access 8, 7615-7621 (2020).

3. Costa F, Borgese M. Systematic design of transmission-type polarization converters comprising multilayered anisotropic metasurfaces. Phys Rev App/ 14, 034049 (2020).

4. Gao YJ, Xiong X, Wang ZH, Chen F, Peng RW et al. Simultaneous generation of arbitrary assembly of polarization states with geometrical-scaling-induced phase modulation. Phys $\operatorname{Rev} X \mathbf{1 0}$, 031035 (2020).

5. Wang S, Deng ZL, Wang YJ, Zhou QB, Wang XL et al. Arbitrary polarization conversion dichroism metasurfaces for all-inone full Poincaré sphere polarizers. Light: Sci Appl 10, 24 (2021).

6. Deng ZL, Deng JH, Zhuang X, Wang S, Li K et al. Diatomic metasurface for vectorial holography. Nano Lett 18, 2885-2892 (2018).

7. Chang CC, Zhao ZX, Li DF, Taylor AJ, Fan SH et al. Broadband linear-to-circular polarization conversion enabled by birefringent off-resonance reflective metasurfaces. Phys Rev Lett 123, 237401 (2019).

8. Ding F, Chang BD, Wei QS, Huang LL, Guan XW et al. Versatile polarization generation and manipulation using dielectric metasurfaces. Laser Photonics Rev 14, 2000116 (2020).

9. Liu J, Shi MQ, Chen Z, Wang SM, Wang ZL et al. Quantum photonics based on metasurfaces. Opto-Electron Adv 4, 200092 (2021).

10. Shalaginov MY, An SS, Yang F, Su P, Lyzwa D et al. Singleelement diffraction-limited fisheye metalens. Nano Lett 20, 7429-7437 (2020).

11. Zentgraf T. Imaging the rainbow. Nat Nanotechnol 13, 179-180 (2018).

12. Balli F, Sultan M, Lami SK, Hastings JT. A hybrid achromatic metalens. Nat Commun 11, 3892 (2020).

13. Rubin NA, D'Aversa G, Chevalier P, Shi ZJ, Chen WT et al. Matrix Fourier optics enables a compact full-Stokes polarization camera. Science 365, eaax1839 (2019).

14. Krasnok A. Metalenses go atomically thick and tunable. Nat Photonics 14, 409-410 (2020).

15. Wang YL, Fan QB, Xu T. Design of high efficiency achromatic metalens with large operation bandwidth using bilayer architecture. Opto-Electron Adv 4, 200008 (2021).

16. Yoon G, Kim K, Huh D, Lee H, Rho J. Single-step manufacturing of hierarchical dielectric metalens in the visible. Nat Commun 11, 2268 (2020).

17. Yoon G, Kim K, Kim SU, Han S, Lee H et al. Printable nanocomposite metalens for high-contrast near-infrared imaging. ACS Nano 15, 698-706 (2021).

18. Moon SW, Kim Y, Yoon G, Rho J. Recent progress on ultrathin metalenses for flat optics. iScience 23, 101877 (2020).

19. Wang JY, Fan JP, Shu H, Liu C, Cheng YZ. Efficiency-tunable terahertz focusing lens based on graphene metasurface. OptoElectron Eng 48, 200319 (2021).

20. Fan JP, Cheng YZ, He B. High-Efficiency ultrathin terahertz geometric metasurface for full-space wavefront manipulation at two frequencies. J Phys D:Appl Phys 54, 115101 (2021).

21. Gao H, Fan XH, Xiong W, Hong MH. Recent advances in optical dynamic meta-holography. Opto-Electron Adv 4, 210030 (2021).

22. Zang $X F$, Ding $H Z$, Intaravanne $Y$, Chen $L$, Peng $Y$ et al. $A$ multi-foci metalens with polarization-rotated focal points. Laser Photonics Rev 13, 1900182 (2019).

23. Fan JP, Cheng YZ. Broadband high-efficiency cross-polarization conversion and multi-functional wavefront manipulation based on chiral structure metasurface for terahertz wave. J Phys D: Appl Phys 53, 025109 (2020).

24. Wang $Q$, Zhang $X Q, X u Y H$, Tian Z, Gu JQ et al. A broadband metasurface-based terahertz flat-lens array. Adv Opt Mater $\mathbf{3}$, 779-785 (2015).

25. Gao S, Park CS, Zhou CY, Lee SS, Choi DY. Twofold polarization-selective all-dielectric trifoci metalens for linearly polarized visible light. Adv Opt Mater 7, 1900883 (2019).

26. Arbabi A, Horie $Y$, Bagheri M, Faraon A. Dielectric metasurfaces for complete control of phase and polarization with subwavelength spatial resolution and high transmission. Nat Nanotechnol 10, 937-943 (2015).

27. Zang XF, Xu WW, Gu M, Yao BS, Chen L et al. Polarization-insensitive metalens with extended focal depth and longitudinal high-tolerance imaging. Adv Opt Mater 8, 1901342 (2020).

28. Li JT, Li J, Zheng CL, Wang SL, Li MY et al. Dynamic control of reflective chiral terahertz metasurface with a new application developing in full grayscale near field imaging. Carbon 172, 189-199 (2021).

29. Xu HX, Hu GW, Li Y, Han L, Zhao JL et al. Interference-assisted kaleidoscopic meta-plexer for arbitrary spin-wavefront manipulation. Light: Sci App/ 8, 3 (2019).

30. Zhou T, Liu Q, Liu YS, Zang XF. Spin-independent metalens for helicity-multiplexing of converged vortices and cylindrical vector beams. Opt Lett 45, 5941-5944 (2020).

31. Wu Z, Wang XK, Sun WF, Feng SF, Han P et al. Vector characterization of zero-order terahertz Bessel beams with linear and circular polarizations. Sci Rep 7, 13929 (2017).

32. Wang XK, Cui Y, Sun WF, Ye JS, Zhang Y. Terahertz real-time imaging with balanced electro-optic detection. Opt Commun 283, 4626-4632 (2010).

33. Li JT, Li J. Terahertz (THz) generator and detection. Electr Sci Eng 2, 11-25 (2020).

\section{Acknowledgements}

This work was supported by the National Natural Science Foundation of China (Nos. 61675147, 61735010 and 91838301), National Key Research and Development Program of China (No. 2017YFA0700202), Basic Research Program of Shenzhen (JCYJ20170412154447469).

\section{Author contributions}

J. T. Li: Conceptualization, Methodology, Writing-Original and Draft preparation. G. C. Wang, Z. Yue: Software and Data curation. J. Y. Liu, J. Li, and C. L. Zheng: Visualization and Investigation. Y. T. Zhang, Y. Zhang and J. Q. Yao: Supervision and Reviewing.

\section{Competing interests}

The authors declare no competing financial interests.

\section{Supplementary information}

Supplementary information is available at https://doi.org/10.29026/oea.2022.210062 